\title{
Design and Baseline Data for a Prospective Observational Study of Rivaroxaban in Patients with Venous Thromboembolism in Japan (XASSENT)
}

\author{
Ikuo Fukuda ${ }^{1, *}$ Atsushi Hirayama ${ }^{2, *}$ Kazuo Kawasugi ${ }^{3}$ Takao Kobayashi ${ }^{4}$ Hideaki Maeda ${ }^{5}$ \\ Mashio Nakamura ${ }^{6}$ Norifumi Nakanishi ${ }^{7}$ Norikazu Yamada ${ }^{8}$ Tsubasa Tajima ${ }^{9}$ Sanghun Iwashiro ${ }^{9}$ \\ Yutaka Okayama ${ }^{10}$ Toshiyuki Sunaya $^{11}$ Kazufumi Hirano ${ }^{10}$ Takanori Hayasaki $^{9}$
}

${ }^{1}$ Department of Cardiovascular Surgery, Suita Tokushukai Hospital, Suita, Japan

${ }^{2}$ Division of Cardiology, Osaka Police Hospital, Osaka, Japan

${ }^{3}$ Department of Internal Medicine, Teikyo University School of Medicine, Tokyo, Japan

${ }^{4}$ Hamamatsu Medical Center, Hamamatsu, Japan

${ }^{5}$ Division of Cardiovascular Surgery, Nihon University School of Medicine, Tokyo, Japan

${ }^{6}$ Nakamura Medical Clinic, Kuwana, Japan

${ }^{7}$ Department of Cardiology, Osaka Namba Clinic, Osaka, Japan

${ }^{8}$ Department of Cardiology, Kuwana City Medical Center, Kuwana, Japan

${ }^{9}$ Medical Affairs Cardiovascular and Nephrology, Medical Affairs and Pharmacovigilance, Bayer Yakuhin, Ltd., Osaka, Japan

10 Pharmacovigilance Monitoring and Governance, Medical Affairs and Pharmacovigilance, Bayer Yakuhin, Ltd., Osaka, Japan

11 Statistics and Data Insights, Data Sciences and Analytics, Research and Development Japan, Bayer Yakuhin, Ltd., Osaka, Japan
Address for correspondence Ikuo Fukuda, MD, PhD, Department of Cardiovascular Surgery, Suita Tokushukai Hospital, 21-1 Senriokanishi, Suita, Osaka 565-0814, Japan (e-mail: ikuofuku@gmail.com).

TH Open 2021;5:e521-e532.

\begin{abstract}
Keywords

- Japan

- pulmonary embolism

- rivaroxaban

- venous thromboembolism

- venous thrombosis
\end{abstract}

Background The efficacy and safety of rivaroxaban have been demonstrated in phase 3 trials of patients with venous thromboembolism (VTE; pulmonary embolism [PE] and deep vein thrombosis [DVT]). Data regarding rivaroxaban treatment of VTE in routine Japanese clinical practice remain limited.

Objectives XASSENT will evaluate rivaroxaban treatment of VTE in real-world Japanese clinical practice. We report the study design and baseline patient characteristics. Methods XASSENT (NCT02558465) is an open-label, prospective observational, postmarketing surveillance cohort study in patients receiving rivaroxaban treatment for VTE. Enrolment took place between November 2015 and March 2018. XASSENT will follow patients for up to 2 years. Primary outcome variables: major bleeding and symptomatic recurrent VTE. Statistical analyses are exploratory and descriptive.

Results Baseline patient characteristics at June $2020(n=2,299)$ are presented (58.2\% female; mean age 66.7 years; mean weight $60.9 \mathrm{~kg}$ ). The population encompasses patients with wide-ranging characteristics including older age, low weight, and

Co-first authors.

received

May 26, 2021

accepted after revision

October 1, 2021
DOI https://doi.org/

10.1055/a-1664-1164.

ISSN 2512-9465. (c) 2021. The Author(s).

This is an open access article published by Thieme under the terms of the Creative Commons Attribution License, permitting unrestricted use, distribution, and reproduction so long as the original work is properly cited. (https://creativecommons.org/licenses/by/4.0/)

Georg Thieme Verlag KG, Rüdigerstraße 14, 70469 Stuttgart, Germany 
renal dysfunction. Most participants (67.6\%) had a history of VTE risk factors at baseline. Half of the population (50.4\%) had DVT only; $41.4 \%$ had DVT with PE; $8.2 \%$ had PE only. Overall, $68.4 \%$ were inpatients and $77.1 \%$ had symptomatic VTE. Rivaroxaban was prescribed for initial treatment in $84.6 \%$ of patients and maintenance treatment in $15.4 \%$. Most were prescribed the approved dose of rivaroxaban for initial ( $30 \mathrm{mg}$ daily; $84.4 \%$ ) or maintenance ( $15 \mathrm{mg}$ daily; $81.9 \%$ ) treatment of VTE in Japan. The most common reason for selecting non-recommended dose was 'elderly'.

Conclusions Results from XASSENT will complement phase 3 trial data and inform clinical practice.

\section{Introduction}

The prevalence of risk factors for venous thromboembolism (VTE; pulmonary embolism [PE] and deep vein thrombosis [DVT]) and the number of VTE diagnoses have increased in Japan in recent decades. ${ }^{1}$ Direct oral anticoagulants, including edoxaban, rivaroxaban, and apixaban, became available for the treatment and prevention of recurrence of VTE in Japan in 2014 and 2015. ${ }^{2}$ There have since been changes in the practice pattern for VTE treatment, with increasing proportions of hospitalized patients who were anticoagulated at discharge after having received direct oral anticoagulants, and decreasing proportions of these patients after receiving warfarin, according to a Japanese claims database study. $^{2}$

Rivaroxaban, an oral, direct factor Xa inhibitor, is suitable for the single-drug treatment of patients with PE or DVT. ${ }^{3,4}$ The efficacy and safety of rivaroxaban have been evaluated in several phase 3 trials of patients with VTE ( - Table 1) ${ }^{5-8}$ In the large, international EINSTEIN-PE and EINSTEIN-DVT trials $(N=8,282)$, a single-drug approach with rivaroxaban had similar efficacy to standard therapy (enoxaparin and warfarin or acenocoumarol) and was associated with a significantly lower rate of major bleeding in patients with symptomatic VTE. ${ }^{8}$ The safety and effectiveness of rivaroxaban in routine clinical practice were then assessed in large, international registries (- Table 1) ${ }^{9,10}$ Pharmacokinetic analyses had shown the exposure of rivaroxaban $15 \mathrm{mg}$ administered to Japanese patients is similar to that of $20 \mathrm{mg}$ to non-Japanese patients. ${ }^{11,12}$ Moreover, target prothrombin time-international normalized ratio (PT-INR) of warfarin for VTE patients is lower in Japan (i.e., PT-INR 1.5 to 2.5 in Japan; 2.0 to 3.0 in western countries). ${ }^{13}$ For these reasons, the smaller J-EINSTEIN-PE and J-EINSTEIN-DVT program $(n=100)$ was performed, in which 15 or $10 \mathrm{mg}$ twice daily followed by $15 \mathrm{mg}$ once daily ( $10 \mathrm{mg}$ twice daily was used only in J-EINSTEIN-DVT) were compared with Japanese standard therapy (unfractionated heparin followed by warfarin) in Japanese patients with symptomatic VTE. ${ }^{5}$ The composite of symptomatic VTE events or asymptomatic deterioration occurred in one patient $(1.3 \%)$ receiving rivaroxaban and in one patient $(5.3 \%)$ receiving unfractionated heparin/warfarin (absolute risk reduction, 4.0\% [95\% confidence interval (CI): $-2.9,24.0]$ ), and there was no major bleeding during study treatment. ${ }^{5}$ Overall, the findings were consistent with those from the international EINSTEIN-PE and EINSTEIN-DVT program. ${ }^{5}$ However, data regarding the effectiveness and safety of rivaroxaban in unselected patients with PE and/or DVT in routine clinical practice in Japan remain limited.

The Xarelto Post-Authorization Safety and Effectiveness Study in Japanese patients with Pulmonary Embolism and/or Deep Vein Thrombosis (XASSENT) is a prospective observational study that will evaluate rivaroxaban in patients with VTE in real-world Japanese clinical practice. This article describes the design of XASSENT and provides baseline data for the study population as of June 2020.

\section{Methods}

\section{Study Design, Objective, and Setting}

XASSENT is an open-label, single-arm, prospective, noninterventional, observational cohort study in patients for whom rivaroxaban treatment for VTE (PE and/or DVT) has been selected (ClinicalTrials.gov identifier: NCT02558465). Its objective is to assess the safety and effectiveness of rivaroxaban for patients with PE and/or DVT in routine clinical use. The study, which is being conducted at multiple medical institutions in Japan, was approved by the Japanese Ministry of Health, Labor and Welfare (MHLW) as a postmarketing surveillance and is being performed in accordance with Good Post-marketing Study Practice standards provided by the MHLW. Separate ethics approval for this postmarketing surveillance study and written informed consent to participate in the surveillance were not required under Japanese regulations, but were obtained when required by a participating center.

The first participant was enrolled in November 2015, after the approval of rivaroxaban for the treatment and prevention of recurrence of VTE in Japan (September 2015), and enrolment continued until March 2018. XASSENT includes a standard observation period of 1 year and will then follow patients for up to 1 year, with data collection taking place at baseline and 1 month, 3 months, 1 year and 2 years after the initiation of rivaroxaban (-Fig. 1). The surveillance is expected to complete on 31 March 2021. 

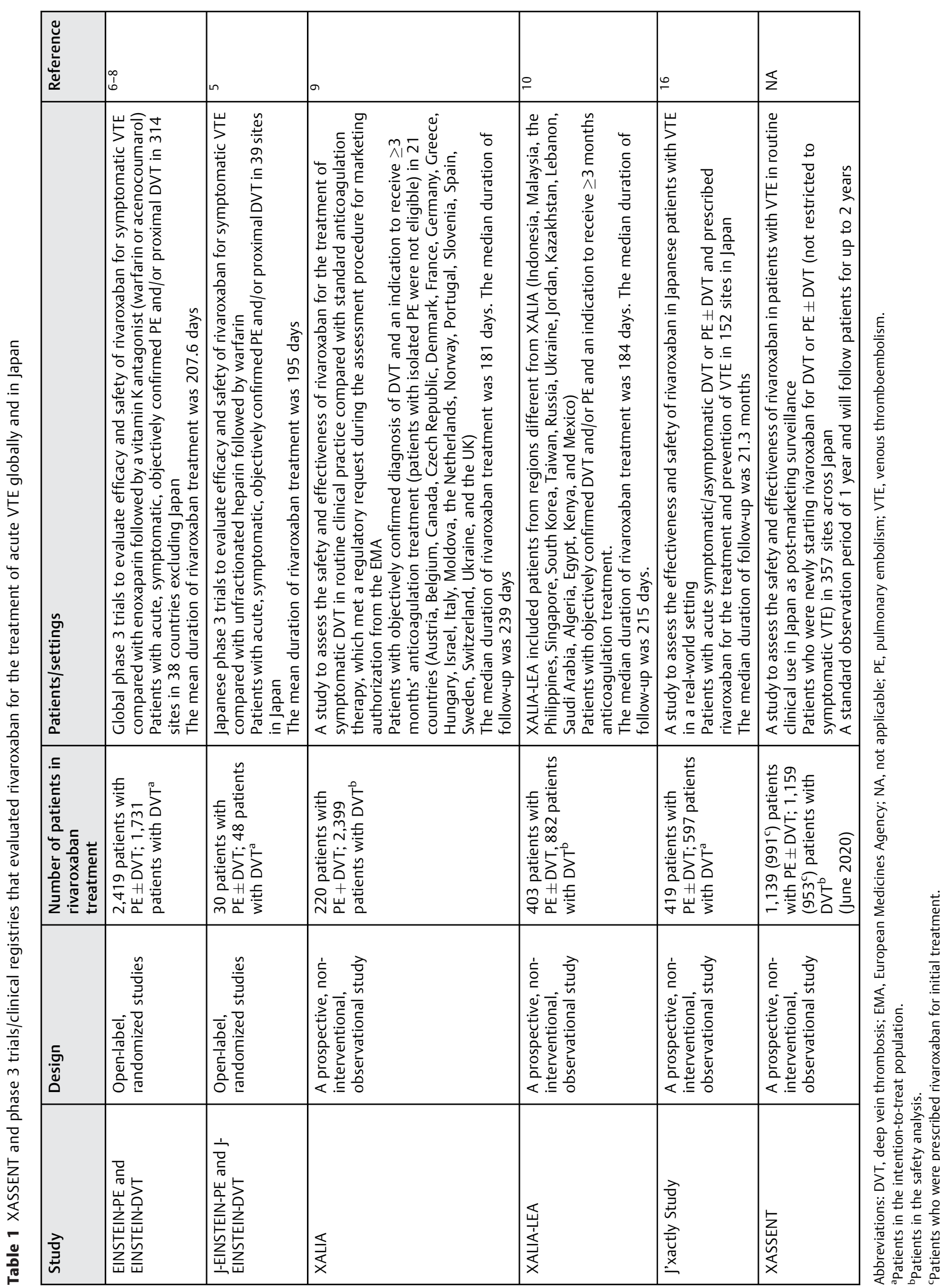


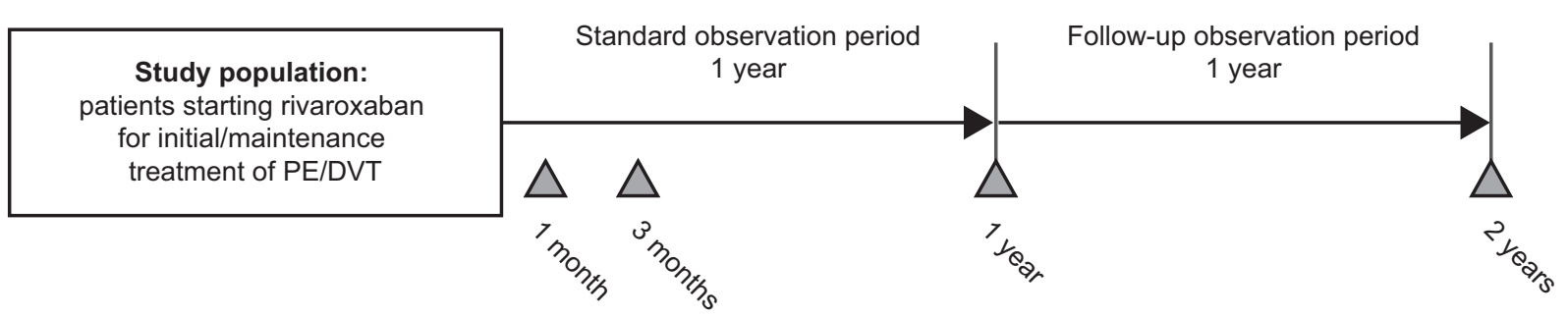

Time of data collection

Fig. 1 Design of XASSENT. DVT, deep vein thrombosis; PE, pulmonary embolism.

\section{Participants}

Consecutive patients who were newly starting rivaroxaban for the treatment and prevention of recurrence of PE/DVT (the index PE/DVT event) were registered for the study by investigators who prescribe rivaroxaban routinely. The diagnosis of PE/DVT was left to attending physicians. The recommendations for PE/DVT diagnosis methods were provided in guidelines by the Japanese Circulation Society Joint Working Group. ${ }^{13}$ Details of diagnosis (such as diagnosis type [DVT only, DVT with PE, PE only], diagnostic method, symptoms, site of occurrence [iliac, femoral, upper extremity etc.]) were recorded on case report forms. The investigator was to have made the choice of treatment (rivaroxaban), in line with the Japanese product label, ${ }^{3}$ before enrolling the patient.

\section{Treatment}

In Japan, rivaroxaban is approved for the treatment and prevention of recurrence of VTE at a dose of $15 \mathrm{mg}$ twice daily ( $30 \mathrm{mg}$ daily) for the first 21 days followed by $15 \mathrm{mg}$ once daily. ${ }^{3}$ The medication is administered orally after meals. No study drug will be provided by the sponsor. If the investigator determines that rivaroxaban treatment should be discontinued or that the patient has been lost to follow-up, surveillance of the patient will be terminated. In case that rivaroxaban is discontinued, post-treatment status and adverse events (only the occurrence of bleeding events and fatal adverse events during follow-up observation period), and PE/DVT recurrence will be recorded for 3 months after rivaroxaban discontinuation, if feasible.

\section{Variables}

Details of the variables assessed during the study are presented in $\boldsymbol{\sim}$ Table $\mathbf{2}$. These variables are reported by attending physicians using case report forms at enrolment and at 1 month, 3 months, 1 year and 2 years after rivaroxaban initiation. Bleeding and recurrent PE/DVT are adjudicated by attending physicians. The primary safety variable is major bleeding, defined in accordance with International Society on Thrombosis and Haemostasis criteria. ${ }^{8}$ Specifically, major bleeding is defined as clinically overt bleeding associated with any of the following: a fatal bleeding; bleeding in a critical area or organ (e.g., intracranial, intraspinal, intraocular, pericardial, intra-articular, retroperitoneal, or intramuscular with compartment syndrome); $\geq 2 \mathrm{~g} / \mathrm{dL}$ reduction in haemoglobin level; or transfusion of $\geq 2$ units of whole blood or packed red blood cells. ${ }^{14}$ The primary effectiveness variable is symptomatic recurrent VTE (a composite of non-fatal or fatal PE or DVT). The diagnosis of recurrent PE/DVT is left to attending physicians, and the details of the diagnosis are recorded on case report forms.

Secondary safety variables include all-cause mortality; vascular events (acute coronary syndrome, ischemic stroke, transient ischemic attack, or systemic embolism); clinically relevant non-major bleeding ${ }^{8}$; and all other adverse events/adverse drug reactions. Secondary effectiveness variables include asymptomatic deterioration of thrombotic burden (D-dimer levels, or imaging test such as computed tomography or complete compression ultrasound) by the end of the standard observation period and distal and/or proximal DVT treatment outcomes.

Bleeding is an event of special interest and will be assessed according to the following categories: major bleeding; clinically relevant non-major bleeding; and minor bleeding. Clinically relevant non-major bleeding is defined as overt bleeding not meeting the criteria for major bleeding, ${ }^{14}$ but requiring medical intervention, an unscheduled visit or telephone call, or interruption/discontinuation of rivaroxaban, or resulting in unpleasant symptoms (e.g., pain) and/or interference with daily life. Minor bleeding is defined as overt bleeding not meeting the definition of major or clinically relevant non-major bleeding.

Other data that will be collected relate to patient characteristics, VTE diagnoses and subtypes, comorbidities, rivaroxaban use, and concomitant therapies ( - Table 2 ).

Historic data (demographic and clinical characteristics) will be collected from the patient's medical records, if available, or by interviewing the patient. If non-recommended dose (i.e., dose other than $30 \mathrm{mg} /$ day for initial treatment and $15 \mathrm{mg} /$ day for maintenance treatments) is selected when initiating rivaroxaban, the reason is recorded on the case report form at 1 month after the initiation of rivaroxaban. All other data required for this study will be collected during routine visits. The end of the standard observation period is 1 year after the start of rivaroxaban treatment, or earlier if rivaroxaban treatment is discontinued, or if the patient discontinues the study (e.g., is withdrawn, lost to follow-up, or dies).

The investigators will use an electronic data capture system to record data from each patient and enter these into a centralized database. 


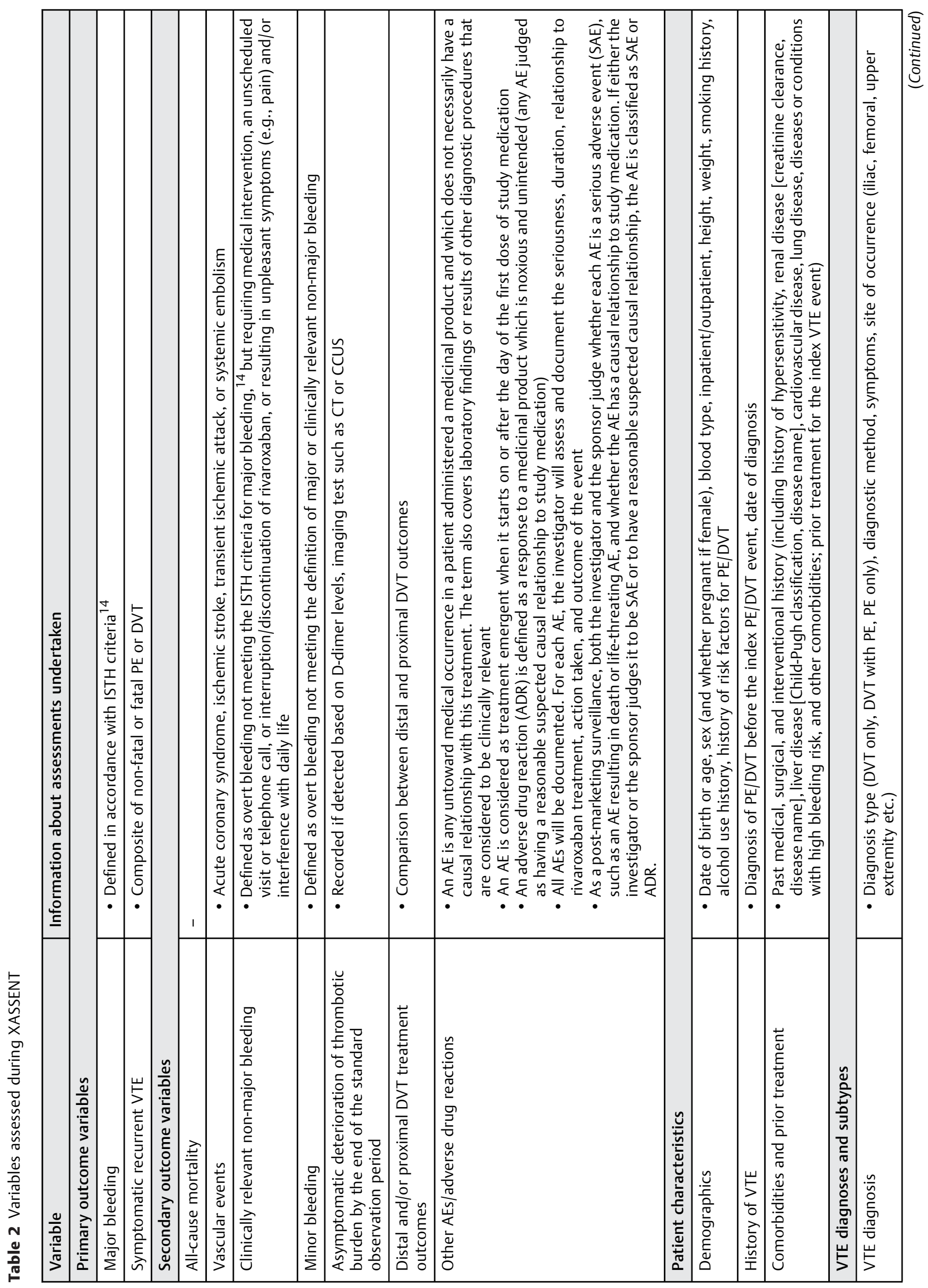




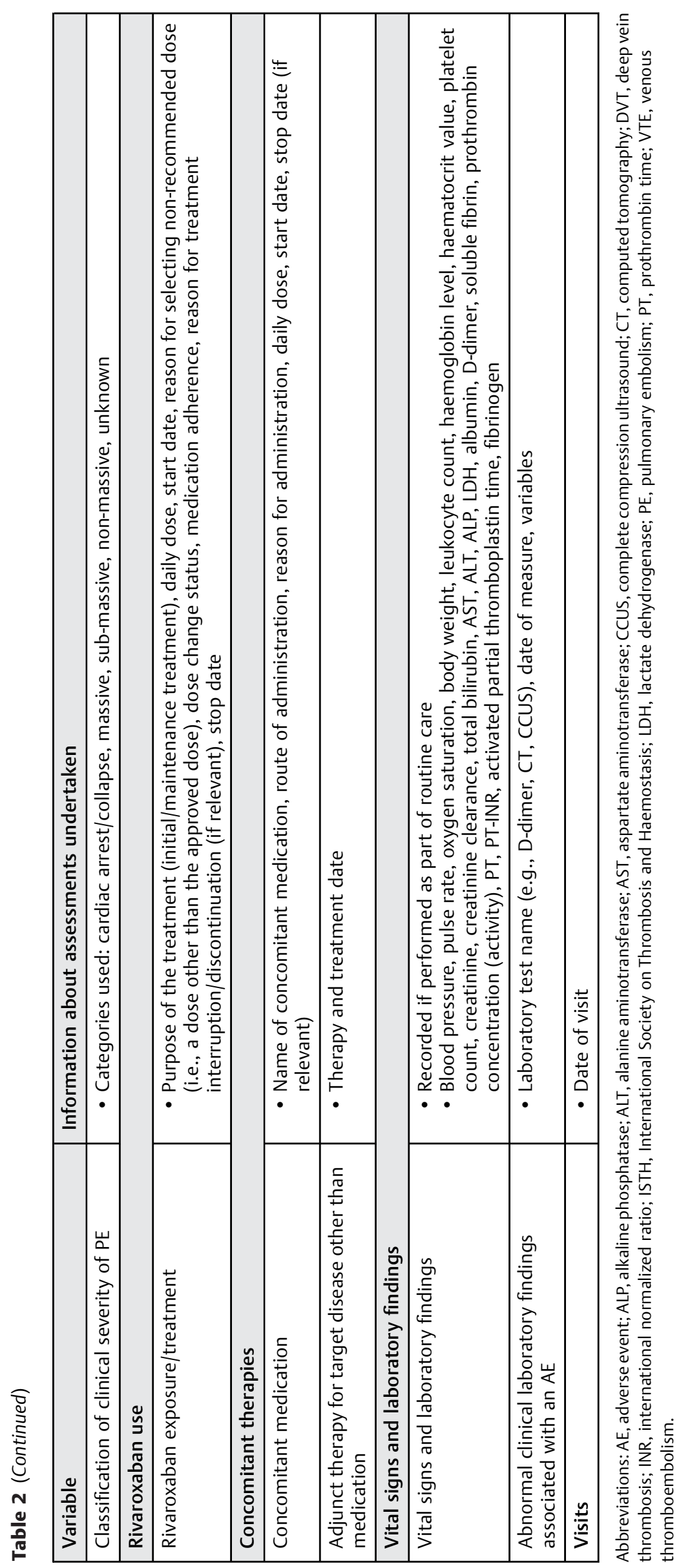




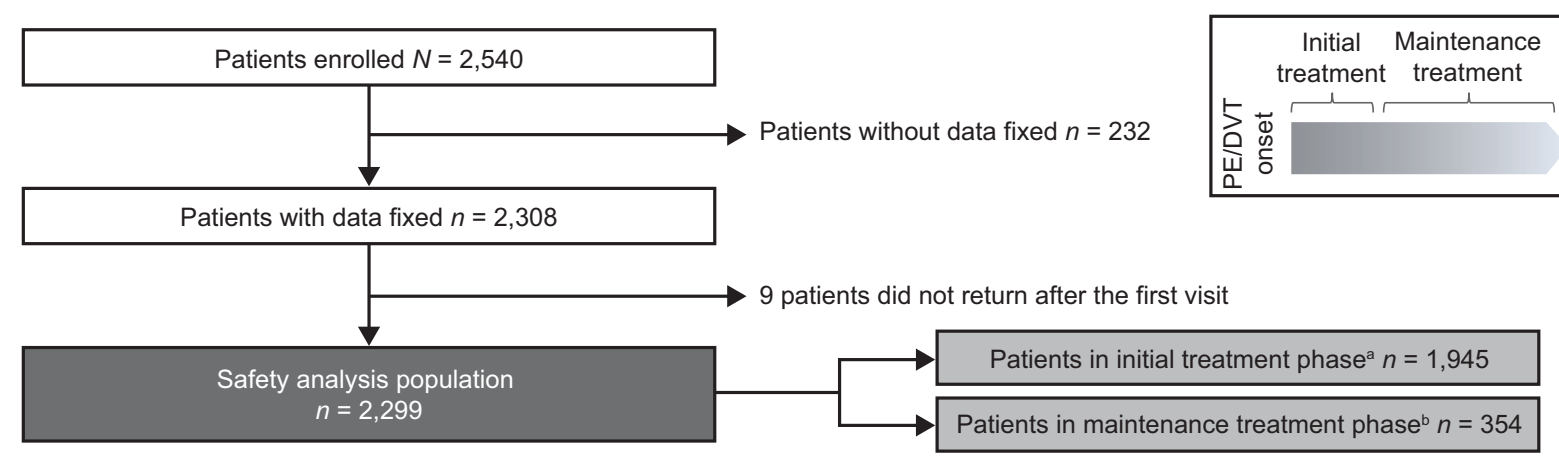

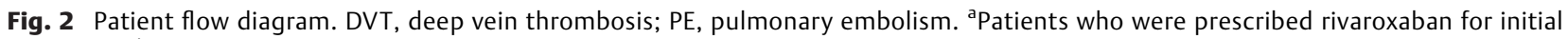
treatment. 'Patients who were prescribed rivaroxaban for maintenance treatment (e.g., switching from other anticoagulants, etc.).

\section{Statistical Methods}

The sample size was determined taking feasibility into account. According to the MHLW 2013 study group data, $\sim 40,000$ patients are estimated to develop VTE (PE or DVT) in Japan each year, with patients with PE accounting for $40 \%$ of a Japanese population with VTE and patients with DVT accounting for the remaining $\sim 60 \%{ }^{15}$ Based on the estimated recruitment rate of sites with an enrolment period of 2.5 years, the sample size was set to $\geq 1,250$. Hemorrhage was the only important identified risk associated with rivaroxaban in the core risk management plan. Based on phase 3 studies, ${ }^{5}$ the expected incidence of any bleeding events was $32.5 \%$. Given these assumptions, among a sample of 1,250 patients, 406 patients would be expected to experience bleeding events during the study, which allows the capture of any bleeding events with a $95 \% \mathrm{CI}$ of $\pm 2.6 \%$. The safety analysis set will include all patients who received at least one dose of rivaroxaban and attended at least one study visit. The effectiveness analysis set will include patients who had PE/DVT diagnosis and were naïve to rivaroxaban at baseline in the safety analysis set.

Data from XASSENT will be analyzed by an independent data center. Statistical analyses are planned to be exploratory and descriptive. Data will be summarized using descriptive statistics (e.g., mean with standard deviation or median with range/interquartile range for continuous variables; frequency for categorical variables). Adverse events will be summarized using the Medical Dictionary for Regulatory Activities coding system. The number of patients with missing data will be presented as a separate category. All statistical analyses will be performed using SAS version 9.4 or higher (SAS Institute Inc., Cary, NC, USA).

For variables of interest, including the primary outcome variables, raw incidence proportion (patients with events/number of treated patients) and incidence rate (patients with events/100 patient-years) will be estimated, together with corresponding 95\% CIs. Time-to-event and multivariate analyses are also planned. In addition, all analyses will be repeated with respect to relevant risk factors and Kaplan-Meier plots will show the time course up to the first event of interest.

Subgroup analyses will be conducted according to age, body weight, renal function, risk factors for VTE (including active cancer [type of cancer, metastasis, chemotherapy]), VTE subtype (PE and/or DVT, clinical severity of PE, symptoms, site/status of the thrombus), purpose of rivaroxaban administration (initial or maintenance treatment), rivaroxaban dose, treatment period (initial treatment, maintenance treatment, after discontinuation), and concomitant therapy.

\section{Results}

Here we report demographics and baseline characteristics for the overall XASSENT population and by purpose of rivaroxaban administration (initial or maintenance treatment) (each as of June 2020). The rivaroxaban doses selected are also presented, together with reasons for choosing a dose other than the approved dose.

XASSENT enrolled 2,540 patients between November 2015 and March 2018 (-Fig. 2). Patients were enrolled in 357 sites across Japan: $52.4 \%$ of the patients were enrolled from study sites with $\geq 400$ beds, $32.8 \%$ from sites with 200 399 beds, $13.2 \%$ from sites with $20-199$ beds, and $1.7 \%$ from sites with $<20$ beds. Baseline patient demographics and clinical characteristics as of June $2020(n=2,299)$ are shown in -Table 3.

Overall, $58.2 \%$ of the XASSENT participants are female. At baseline, the mean age was 66.7 years, the mean body weight was $60.9 \mathrm{~kg}$, and the mean creatinine clearance was 83.0 $\mathrm{mL} / \mathrm{min}$ (-Table 3 ). However, the study population encompasses patients with a wide range of characteristics, including elderly patients, individuals with a low body weight, and patients with renal dysfunction (as assessed by creatinine clearance) (-Table 3). Most of the participants (67.6\%) had a history of risk factors for VTE at baseline, with active cancer reported for 386 patients (16.8\%) (-Table 3). Cardiopulmonary disease was reported as a risk factor for VTE for 140 patients (6.1\%). Half of the XASSENT population (50.4\%) had a diagnosis of DVT only, with $16.9 \%$ overall having isolated distal DVT; 41.4\% had DVT with PE, and $8.2 \%$ had PE only (of varying severity) (-Table 3). Approximately two-thirds of the participants $(68.4 \%)$ were inpatients. Most patients (77.1\%) had symptomatic VTE, but patients with asymptomatic VTE were also represented (-Table $\mathbf{3}$ ).

The purpose of rivaroxaban administration was initial treatment in 1,945 patients (84.6\%) and maintenance 
Table 3 Demographics and clinical characteristics of XASSENT participants at baseline

\begin{tabular}{|c|c|c|c|}
\hline \multirow[t]{2}{*}{ Characteristic } & \multicolumn{2}{|c|}{ Purpose of rivaroxaban administration } & \multirow[t]{2}{*}{ Total $(n=2,299)$} \\
\hline & $\begin{array}{l}\text { Initial treatment } \\
(n=1,945)\end{array}$ & $\begin{array}{l}\text { Maintenance } \\
\text { treatment }(n=354)\end{array}$ & \\
\hline Age, years & $66.5 \pm 15.2$ & $67.6 \pm 14.0$ & $66.7 \pm 15.0$ \\
\hline \multicolumn{4}{|l|}{ Age category, years } \\
\hline$<65$ & $697(35.8)$ & $122(34.5)$ & $819(35.6)$ \\
\hline 65 to $<75$ & $572(29.4)$ & $98(27.7)$ & $670(29.1)$ \\
\hline$\geq 75$ & $676(34.8)$ & $134(37.9)$ & $810(35.2)$ \\
\hline Female & $1,115(57.3)$ & $223(63.0)$ & $1,338(58.2)$ \\
\hline Body weight, kg & $61.3 \pm 13.9$ & $58.7 \pm 13.7$ & $60.9 \pm 13.9$ \\
\hline \multicolumn{4}{|l|}{ Body weight category, kg } \\
\hline$>50$ & $1,459(75.0)$ & $252(71.2)$ & $1,711(74.4)$ \\
\hline$\leq 50$ & $418(21.5)$ & $90(25.4)$ & $508(22.1)$ \\
\hline Unknown & $68(3.5)$ & $12(3.4)$ & $80(3.5)$ \\
\hline $\mathrm{BMI}^{\mathrm{a}}$ & $24.0 \pm 4.2$ & $23.5 \pm 4.4$ & $24.0 \pm 4.2$ \\
\hline Creatinine clearance, $\mathrm{mL} / \mathrm{min}$ & $83.8 \pm 37.1$ & $79.0 \pm 35.1$ & $83.0 \pm 36.8$ \\
\hline \multicolumn{4}{|l|}{ Creatinine clearance category, mL/min } \\
\hline$<30$ & $9(0.5)$ & $2(0.6)$ & $11(0.5)$ \\
\hline 30 to $<50$ & $273(14.0)$ & 60 (16.9) & $333(14.5)$ \\
\hline 50 to $<80$ & $738(37.9)$ & $141(39.8)$ & $879(38.2)$ \\
\hline$\geq 80$ & $851(43.8)$ & $137(38.7)$ & $988(43.0)$ \\
\hline Unknown & $74(3.8)$ & $14(4.0)$ & $88(3.8)$ \\
\hline Inpatients & $1,417(72.9)$ & $156(44.1)$ & $1,573(68.4)$ \\
\hline Outpatients & $528(27.1)$ & $198(55.9)$ & $726(31.6)$ \\
\hline \multicolumn{4}{|l|}{ VTE diagnosis } \\
\hline DVT only & $953(49.0)$ & $206(58.2)$ & $1,159(50.4)$ \\
\hline Isolated distal DVT & $323(16.6)$ & $65(18.4)$ & $388(16.9)$ \\
\hline DVT other than isolated distal & $611(31.4)$ & $111(31.4)$ & $722(31.4)$ \\
\hline Unidentifiable & $0(0.0)$ & $3(0.8)$ & $3(0.1)$ \\
\hline Unknown & $19(1.0)$ & $27(7.6)$ & $46(2.0)$ \\
\hline PE only & $150(7.7)$ & $38(10.7)$ & $188(8.2)$ \\
\hline PE with DVT & $841(43.2)$ & $110(31.1)$ & $951(41.4)$ \\
\hline \multicolumn{4}{|l|}{ Clinical severity of PE } \\
\hline Cardiac arrest/collapse & $9(0.5)$ & $5(1.4)$ & $14(0.6)$ \\
\hline Massive & $59(3.0)$ & $7(2.0)$ & $66(2.9)$ \\
\hline Sub-massive & $306(15.7)$ & $37(10.5)$ & $343(14.9)$ \\
\hline Non-massive & $586(30.1)$ & $76(21.5)$ & $662(28.8)$ \\
\hline Unknown & $31(1.6)$ & $23(6.5)$ & $54(2.3)$ \\
\hline Other & $1(0.1)$ & $0(0.0)$ & $1(<0.1)$ \\
\hline \multicolumn{4}{|l|}{ VTE symptoms } \\
\hline Symptomatic PE/DVT & $1,551(79.7)$ & $221(62.4)$ & $1,772(77.1)$ \\
\hline Asymptomatic PE/DVT & $393(20.2)$ & $133(37.6)$ & $526(22.9)$ \\
\hline History of PE/DVT & $183(9.4)$ & $98(27.7)$ & $281(12.2)$ \\
\hline History of risk factors for VTE ${ }^{b}$ & $1,320(67.9)$ & $233(65.8)$ & $1,553(67.6)$ \\
\hline Immobilization within 3 months & $366(18.8)$ & $61(17.2)$ & $427(18.6)$ \\
\hline
\end{tabular}


Table 3 (Continued)

\begin{tabular}{|c|c|c|c|}
\hline \multirow[t]{2}{*}{ Characteristic } & \multicolumn{2}{|c|}{ Purpose of rivaroxaban administration } & \multirow[t]{2}{*}{ Total $(n=2,299)$} \\
\hline & $\begin{array}{l}\text { Initial treatment } \\
(n=1,945)\end{array}$ & $\begin{array}{l}\text { Maintenance } \\
\text { treatment }(n=354)\end{array}$ & \\
\hline Surgery/injury within 3 months & $379(19.5)$ & $64(18.1)$ & $443(19.3)$ \\
\hline Obesity & $355(18.3)$ & $44(12.4)$ & $399(17.4)$ \\
\hline Active cancer & $312(16.0)$ & $74(20.9)$ & $386(16.8)$ \\
\hline \multicolumn{4}{|l|}{ Comorbidities other than risk factors for PE/DVT } \\
\hline Renal disease & $173(8.9)$ & $39(11.0)$ & $212(9.2)$ \\
\hline Liver disease & $128(6.6)$ & $37(10.5)$ & $165(7.2)$ \\
\hline Cardiovascular disease & $900(46.3)$ & $174(49.2)$ & $1,074(46.7)$ \\
\hline Hypertension & $783(40.3)$ & $134(37.9)$ & $917(39.9)$ \\
\hline Atrial fibrillation & $78(4.0)$ & $17(4.8)$ & $95(4.1)$ \\
\hline Lung disease & $193(9.9)$ & $37(10.5)$ & $230(10.0)$ \\
\hline Diseases or conditions with high risk of bleeding ${ }^{c}$ & $306(15.7)$ & $63(17.8)$ & $369(16.1)$ \\
\hline Use of antiplatelet drugs $^{\mathrm{d}}$ & $154(7.9)$ & $42(11.9)$ & $196(8.5)$ \\
\hline \multicolumn{4}{|l|}{ Anticoagulation therapy other than rivaroxaban } \\
\hline $\begin{array}{l}\text { Anticoagulation for the index PE/DVT } \\
\text { (initial treatment only) }\end{array}$ & $441(22.7)$ & - & - \\
\hline $\begin{array}{l}\text { Anticoagulation within } 3 \text { months before the initiation } \\
\text { of rivaroxaban (maintenance treatment only) }\end{array}$ & - & $228(64.4)$ & - \\
\hline Unfractionated heparin & $377(19.4)$ & $97(27.4)$ & - \\
\hline Fondaparinux & $2(0.1)$ & $0(0.00)$ & - \\
\hline Warfarin & $44(2.3)$ & $111(31.4)$ & - \\
\hline Other & $129(6.6)$ & $64(18.1)$ & - \\
\hline Use of inferior vena cava filter & $116(6.0)$ & - & - \\
\hline
\end{tabular}

Abbreviations: BMI, body mass index; DVT, deep vein thrombosis; PE, pulmonary embolism; VTE, venous thromboembolism.

Data cut-off: June 2020. Data are presented as mean \pm standard deviation or as $n(\%)$.

${ }^{\mathrm{a} B M I}$ missing for 191 patients (163 for initial treatment and 28 for maintenance treatment).

${ }^{\mathrm{b}}$ Risk factors reported in $\geq 10 \%$ of total patients are listed.

'Includes haemostasis or coagulation disorders, congenital or acquired haemorrhagic disorders, uncontrollable severe hypertension, vascular retinopathy, active cancer, active ulcerative gastrointestinal disorders, short days after the onset of gastrointestinal ulcers, short days after the onset of intracranial hemorrhage, vascular abnormalities in the spinal cord or brain, short days after cerebral spinal cord or eye surgery, and history of bronchiectasis or pulmonary hemorrhage.

${ }^{\mathrm{d} D u r i n g}$ observational periods.

treatment in 354 patients $(15.4 \%)$ ( - Table 3 and - Fig. 3 ). The mean creatinine clearance was $83.8 \mathrm{~mL} / \mathrm{min}$ in patients receiving rivaroxaban as initial treatment, while it was $79.0 \mathrm{~mL} / \mathrm{min}$ in those receiving rivaroxaban as maintenance treatment ( - Table 3 ). Among patients in the initial treatment group, $72.9 \%$ were inpatients, $79.7 \%$ had symptomatic VTE, and $49.0 \%$ had DVT only. Those were respectively $44.1 \%$, $62.4 \%$, and $58.2 \%$ among those in the maintenance treatment group ( - Table 3). In the maintenance treatment group, 7.6\% of patients had DVT only with an unknown site (-Table 3 ). Obesity and a history of VTE were reported in $18.3 \%$ and $9.4 \%$ of patients in the initial treatment group, while those were $12.4 \%$ and $27.7 \%$ in the maintenance treatment group (-Table 3). In the initial treatment group, $22.7 \%$ of patients received anticoagulation other than rivaroxaban for the index PE/DVT (mainly unfractionated heparin). Inferior vena cava filter was placed in $6.0 \%$ of patients ( - Table 3 ), and low proportions of patients underwent thrombolysis, thrombectomy, or catheter-assisted thrombus removal (fragmentation or aspiration thrombectomy) (4.4\%, $0.1 \%$, and $0.8 \%$, respectively). In the maintenance treatment group, $64.4 \%$ of patients received unfractionated heparin, warfarin, and other anticoagulants (except fondaparinux) in the 3 months before the initiation of rivaroxaban ( - Table 3 ).

Around $80 \%$ of patients were prescribed the dose of rivaroxaban approved for the initial or maintenance treatment of VTE in Japan (-Fig. 3). The most common reason for selecting non-recommended dose (i.e., other than 30 $\mathrm{mg} /$ day in initial treatment or $15 \mathrm{mg} /$ day in maintenance treatment) was that the patient was elderly (-Fig. 3). Other reasons included non-severe/non-acute VTE, the presence of renal dysfunction, bleeding risk (judged by attending physicians), low body weight, and the use of a concomitant drug (-Fig. 3). 
A

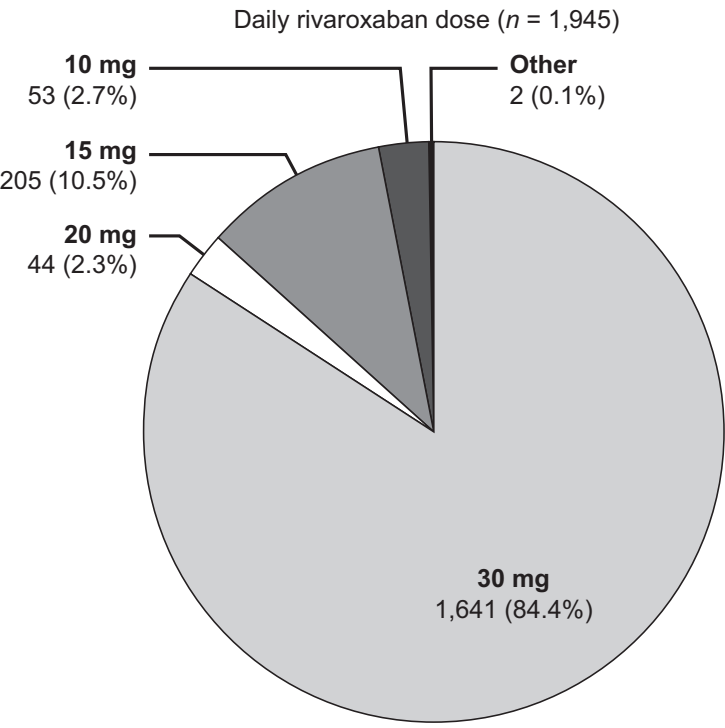

\begin{tabular}{lc}
$\begin{array}{l}\text { Reasons for selecting the } \\
\text { dose other than } \mathbf{3 0} \mathbf{~ m g} \\
\text { (multiple choice allowed) }\end{array}$ & $\begin{array}{c}\text { Patients } \\
(\boldsymbol{n}=\mathbf{3 0 4})\end{array}$ \\
\hline Elderly & 100 \\
Not severe VTE/non-acute phase & 73 \\
Renal dysfunction & 51 \\
Bleeding risk & 46 \\
Low body weight & 38 \\
Concomitant drug & 15 \\
Other & 52 \\
\hline
\end{tabular}

B

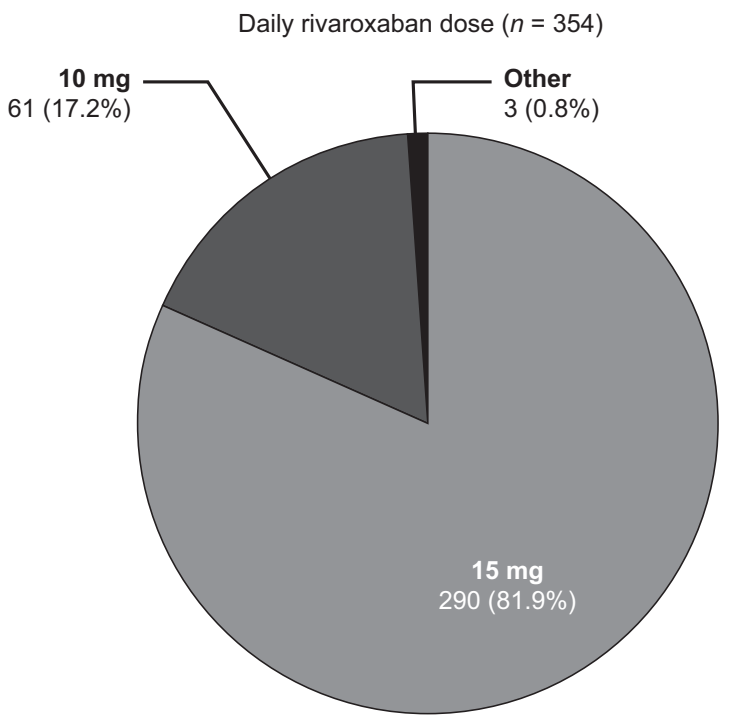

\begin{tabular}{lc}
$\begin{array}{l}\text { Reasons for selecting the } \\
\text { dose other than } \mathbf{1 5} \mathbf{~ m g} \\
\text { (multiple choice allowed) }\end{array}$ & $\begin{array}{c}\text { Patients } \\
(\boldsymbol{n}=\mathbf{6 4})\end{array}$ \\
\hline Elderly & 28 \\
Renal dysfunction & 14 \\
Low body weight & 10 \\
Bleeding risk & 7 \\
Not severe VTE/non-acute phase & 5 \\
Concomitant drug & 1 \\
Other & 13 \\
\hline
\end{tabular}

Fig. 3 Rivaroxaban doses selected for (A) initial treatment and (B) maintenance treatment in XASSENT participants, with reasons for selecting non-recommended dose (i.e., a dose other than the approved dose). Data are presented as $n$ (\%) or as $n$. VTE, venous thromboembolism. Note: The reasons for the dose selection described by attending physicians were categorized and tabulated. Bleeding risk was a judgement by attending physicians. Reasons for a patient who received $15 \mathrm{mg}$ in the initial treatment group are missing.

\section{Discussion}

XASSENT is evaluating the safety and effectiveness of rivaroxaban in patients with VTE in routine Japanese clinical practice. These results will provide real-world evidence that may complement data from the phase $3 \mathrm{~J}$-EINSTEIN-PE and JEINSTEIN-DVT trials. ${ }^{5}$ In total, 2,540 patients with a broad range of characteristics, VTE subtypes, and comorbidities have been enrolled and will be followed for up to 2 years. XASSENT will provide incidence data for multiple variables, with a focus on bleeding and recurrent VTE events. The design of the study allows the collection of detailed information related to exposure, minimizes recall bias, and provides information on the timing of events relative to rivaroxaban administration. Exploratory subgroup analyses are planned to provide information for patient groups of interest.
The J'xactly Study ${ }^{16}$ is another prospective observational study conducted in 1,039 Japanese patients with VTE who were prescribed rivaroxaban ( - Table 1 ). Baseline characteristics were generally comparable between the two studies, although the J'xactly Study included higher proportions of outpatients (41.5\%), patients with DVT only (58.8\%), and patients with creatinine clearance $<50 \mathrm{~mL} / \mathrm{min}(22.4 \%) .{ }^{16}$ In the J'xactly Study, the incidence of recurrence or aggravation of symptomatic VTE was $2.6 \%$ per patient-year and the incidence of International Society on Thrombosis and Haemostasis major bleeding was $2.9 \%$ per patient-year. ${ }^{16} \mathrm{~A}$ single-drug approach with rivaroxaban was shown to be a valuable treatment for a wide range of patients with VTE in Japanese clinical practice. ${ }^{16}$ However, more real-world data are required to support the single-drug approach with rivaroxaban for Japanese patients with VTE. In the J'xactly 
Study, $65.6 \%$ of patients received an initial rivaroxaban dose of $30 \mathrm{mg}$ daily included in the Japanese product label. ${ }^{16}$ In contrast, $84.4 \%$ of XASSENT participants received an initial daily rivaroxaban dose of $30 \mathrm{mg}$, with lower proportions receiving other doses owing to patient-related factors (e.g., older age).

Safety and effectiveness data from patients who were not well represented in phase 3 trials or the XALIA non-interventional study of rivaroxaban, such as patients with asymptomatic VTE ( - Table 1) $)^{5,8-10}$ will help to inform unmet needs related to the management of VTE. XASSENT will provide further evidence regarding patients with VTE in Japan, particularly for patients who are elderly or who have a low body weight, renal dysfunction, or active cancer. $^{5,8,17}$ For example, $17 \%$ of XASSENT participants had active cancer at baseline, compared with $6 \%$ of the rivaroxaban groups in the EINSTEIN-PE and EINSTEIN-DVT trials. ${ }^{17}$ Patients with cancer and venous thrombosis are more likely to develop recurrent thromboembolism ( $\sim 4$ times) and major bleeding ( $\sim 2$ times) during anticoagulant treatment than those without cancer. ${ }^{18}$

XASSENT is one of the largest real-world observational studies of VTE treatment and prevention in Japan. It will add to evidence from other Japanese real-world studies of VTE management, such as the Japan VTE Treatment Registry $(J A V A)^{19}$ or COMMAND VTE Registry, ${ }^{20}$ which mainly enrolled patients before the introduction of direct oral anticoagulants for VTE in Japan, and the Edoxaban Treatment in routine cliNical prActice in patients with Venous ThromboEmbolism - Japan (ETNA-VTE-Japan) study of edoxaban. ${ }^{21}$

Open-label, single-arm observational studies such as XASSENT have inherent limitations, including the possibility of selection bias (e.g., arising from the investigators' choices in routine clinical practice), confounding variables (e.g., dose of rivaroxaban used chosen at discretion of attending physicians), loss of patients to follow-up that may result in underestimation of incidence of clinical events evaluated by attending physicians, and the lack of mandatory laboratory tests. Furthermore, no formal diagnosis methods or criteria for diagnosis of VTE are specified in this study. However, the recommendations for the diagnosis methods were provided in guidelines, ${ }^{13}$ and the diagnosis methods used and clinical presentations (e.g., symptoms, site of occurrence) are recorded on case report forms by attending physicians. The study is not powered to evaluate rare events.

In conclusion, XASSENT has enrolled 2,540 patients with VTE who are being treated with rivaroxaban and is one of the largest real-world observational studies of VTE management in Japan. XASSENT participants have a broad range of characteristics and comorbidities, representing real-world patients with VTE. XASSENT will provide real-world information on the safety and effectiveness of rivaroxaban for VTE treatment in routine Japanese care, to complement data from phase 3 trials and inform clinical practice.

\section{Addendum}

I.F., A.H., K.K., T.K., H.M., M.N., N.N., N.Y., Y.O. and T.S. contributed to developing protocol, study execution, data analysis, interpretation of results, and editing the manuscript. T.T., S.I., K.H., T.H. contributed to data analysis, interpretation of the results, and drafting/editing the manuscript. The manuscript has been read and approved for submission to the TH Open by all authors.

\section{Prior Presentation}

None.

\section{Funding}

Bayer Yakuhin, Ltd.

\section{Conflict of Interest}

IF has received payment or honoraria from Bayer Yahukin Ltd., Daiichi Sankyo Co. Ltd. and Pfizer. AH has received grants or contracts from Boston Scientific Japan K.K., Fukuda Denshi Co., Ltd., St. Jude Medical Co., Ltd., Medtronic Japan Co., Ltd. and Japan Lifeline Co., Ltd, and payment or honoraria from TOA EIYO Ltd., Nippon Boehringer Ingelheim Co., Ltd., Amgen Astellas BioPharma K.K., Sanofi K.K., Astellas Pharma Inc., Sumitomo Dainippon Pharma Co., Ltd., Bristol-Myers Squibb K.K., AstraZeneca K.K., Daiichi Sankyo Co., Ltd., Bayer Yakuhin, Ltd. KK has received payment or honoraria from Bayer Yahukin Ltd. MN has received payment or honoraria from Daiichi Sankyo Co., Ltd.. NY has received payment or honoraria from Bayer Yakuhin, Ltd., Pfizer Japan Inc., Daiichi Sankyo Co., Ltd. TK, HM and NN have no conflict of interest. TT, SI, YO, TS, KH and TH are employees of Bayer Yakuhin, Ltd.

\section{Acknowledgments}

This study was sponsored by Bayer. The authors thank Fujitsu Japan Limited, Tokyo, Japan for EDC system and electronic CRF set-up, EPS Corporation, Tokyo, Japan for data management and analysis, and Oxford PharmaGenesis, Oxford, UK for providing medical writing support, which were funded by Bayer Yakuhin, Ltd., Osaka, Japan.

\section{References}

1 Nakamura M, Yamada N, Ito M. Current management of venous thromboembolism in Japan: Current epidemiology and advances in anticoagulant therapy. J Cardiol 2015;66(06):451-459

2 Yamashita Y, Morimoto T, Yoshikawa Y, et al. Temporal Trends in the Practice Pattern for Venous Thromboembolism in Japan: Insight From JROAD-DPC. J Am Heart Assoc 2020;9(02):e014582

3 Bayer Yakuhin Ltd. Package insert for Xarelto tablets $10 \mathrm{mg} / 15 \mathrm{mg}$. https://www.pmda.go.jp/PmdaSearch/iyakuDetail/ResultDataSetPDF/ 630004_3339003F1024_1_20. Accessed April 13, 2021

4 European Medicines Agency. XARELTO ${ }^{\circ}$ (rivaroxaban). https:// www.ema.europa.eu/en/medicines/human/EPAR/xarelto. Published 2020. Accessed 06 May 2021

5 Yamada N, Hirayama A, Maeda $H$, et al. Oral rivaroxaban for Japanese patients with symptomatic venous thromboembolism the J-EINSTEIN DVT and PE program. Thromb J 2015;13:2

6 Bauersachs R, Berkowitz SD, Brenner B, et al; EINSTEIN Investigators. Oral rivaroxaban for symptomatic venous thromboembolism. N Engl J Med 2010;363(26):2499-2510 
7 Büller HR, Prins MH, Lensin AW, et al; EINSTEIN-PE Investigators. Oral rivaroxaban for the treatment of symptomatic pulmonary embolism. N Engl J Med 2012;366(14):1287-1297

8 Prins MH, Lensing AWA, Bauersachs R, et al; EINSTEIN Investigators. Oral rivaroxaban versus standard therapy for the treatment of symptomatic venous thromboembolism: a pooled analysis of the EINSTEIN-DVT and PE randomized studies. Thromb J 2013;11(01):21

9 Ageno W, Mantovani LG, Haas S, et al. Safety and effectiveness of oral rivaroxaban versus standard anticoagulation for the treatment of symptomatic deep-vein thrombosis (XALIA): an international, prospective, non-interventional study. Lancet Haematol 2016;3(01):e12-e21

10 Kreutz R, Mantovani LG, Haas S, et al. XALIA-LEA: An observational study of venous thromboembolism treatment with rivaroxaban and standard anticoagulation in the Asia-Pacific, Eastern Europe, the Middle East, Africa and Latin America. Thromb Res 2019;176:125-132

11 Tanigawa T, Kaneko M, Hashizume K, et al. Model-based dose selection for phase III rivaroxaban study in Japanese patients with non-valvular atrial fibrillation. Drug Metab Pharmacokinet 2013; 28(01):59-70

12 Hori M, Matsumoto M, Tanahashi N, et al; J-ROCKET AF study investigators. Rivaroxaban vs. warfarin in Japanese patients with atrial fibrillation - the J-ROCKET AF study -. Circ J 2012;76(09): 2104-2111

13 JCS Joint Working Group. Guidelines for the diagnosis, treatment and prevention of pulmonary thromboembolism and deep vein thrombosis (JCS 2009). Circ J 2011;75(05):1258-1281

14 Schulman S, Kearon CSubcommittee on Control of Anticoagulation of the Scientific and Standardization Committee of the International Society on Thrombosis and Haemostasis. Definition of major bleeding in clinical investigations of antihemostatic medicinal products in non-surgical patients. J Thromb Haemost 2005;3(04):692-694

15 Ota S, Matsuda A, Ogihara Y, et al. Incidence, Characteristics and Management of Venous Thromboembolism in Japan During 2011. Circ J 2018;82(02):555-560

16 Okumura Y, Fukuda I, Nakamura M, et al; J'xactly Investigators. A Multicenter Prospective Observational Cohort Study to Investigate the Effectiveness and Safety of Rivaroxaban in Japanese Venous Thromboembolism Patients (The J'xactly Study). Circ J 2020;84(11):1912-1921

17 Prins MH, Lensing AW, Brighton TA, et al. Oral rivaroxaban versus enoxaparin with vitamin $\mathrm{K}$ antagonist for the treatment of symptomatic venous thromboembolism in patients with cancer (EINSTEIN-DVT and EINSTEIN-PE): a pooled subgroup analysis of two randomised controlled trials. Lancet Haematol 2014;1(01): e37-e46

18 Prandoni P, Lensing AW, Piccioli A, et al. Recurrent venous thromboembolism and bleeding complications during anticoagulant treatment in patients with cancer and venous thrombosis. Blood 2002;100(10):3484-3488

19 Nakamura M, Miyata T, Ozeki Y, et al. Current venous thromboembolism management and outcomes in Japan. Circ J 2014;78 (03):708-717

20 Yamashita Y, Morimoto T, Amano $\mathrm{H}$, et al; COMMAND VTE Registry Investigators. Anticoagulation Therapy for Venous Thromboembolism in the Real World - From the COMMAND VTE Registry. Circ J 2018;82(05):1262-1270

21 Nakamura M, Yamada N, Asamura T, Shiosakai K, Uchino K. Safety and Effectiveness of Edoxaban in Japanese Venous Thromboembolism Patients - Final Analysis of One-Year Follow-up Data From a Japanese Postmarketing Observational Study (ETNA-VTEJapan). Circ Rep 2020;2(03):192-202 